\title{
Nonlocal noise cross correlation mediated by entangled Majorana fermions
}

\author{
Hai-Feng Lü, Hai-Zhou Lu, and Shun-Qing Shen \\ Department of Physics, The University of Hong Kong, Pokfulam Road, Hong Kong, China \\ (Received 25 April 2012; revised manuscript received 30 July 2012; published 27 August 2012)
}

\begin{abstract}
Due to their nonlocality, qubits nested in Majorana bound states may be the key to realize decoherence-free quantum computation. Majorana bound states could be achieved at the ends of a one-dimensional topological superconductor. However, when the bound states couple directly to electron reservoirs their nonlocal correlation is quenched by local Andreev reflections. Here we propose a scheme to generate nonlocal noise cross correlation between two well-separated quantum dots, mediated by a pair of Majorana bound states. Both positive and negative cross correlations can be obtained by tuning the gate voltages applied to the dots. Within a limited range of finite temperatures, the cross correlation is not suppressed by thermal fluctuations. Furthermore, we show how the local Andreev reflections suppress the noise cross correlation when multiple dot energy levels are coupled to the Majorana bound states. The measurable cross correlation is expected to serve as a sensitive indicator for the generation of Majorana fermions.
\end{abstract}

DOI: 10.1103/PhysRevB.86.075318

PACS number(s): 72.70.+m, 03.75.Lm, 73.21.La, 74.78.Na

\section{INTRODUCTION}

Majorana fermions are exotic because they are their own antiparticles. ${ }^{1}$ The search for Majorana fermions and controlling of Majorana bound states in solid-state devices are currently attracting increasing attention due to their potential application in quantum information..$^{2-9}$ Two wellseparated Majorana bound states can define a nonlocal fermion level and its occupation encodes a qubit. This nonlocal topological qubit is robust against decoherence from local perturbations or interactions. ${ }^{2-4}$ So far, a number of schemes have been proposed to realize and to detect the Majorana bound states. ${ }^{10-20}$ For instance, it was discovered that the Majorana bound states can be realized at the ends of a semiconductor nanowire with strong spin-orbit interaction in the proximity of an $s$-wave superconductor. ${ }^{10-12}$ Very recently, the signature for the formation of a spatially separated pair of Majorana bound states have been observed experimentally in such a system, in terms of a zero-bias conductance peak..$^{21,22}$ However, the existence of Majorana fermions is still controversial because the zero-bias conductance enhancement could also be the signature of diverse phenomena in mesoscopic physics. ${ }^{23,24}$

A unique feature of Majorana bound states is that their superposition can form a nonlocal fermion level. Based on this nonlocality, the nonlocal current-current correlation in tunneling measurements would provide supporting evidence. Actually, the current noise cross correlation can reveal information related to particle fluctuations and is a powerful tool for studying different types of interactions and quantum statistics. ${ }^{25}$ For free particles, fermions tend to induce negative noise cross correlation while bosons tend to induce positive one due to quantum statistics of indistinguishable identical particles. ${ }^{26}$ In mesoscopic systems, positive cross correlation can be induced in the presence of Bardeen-Cooper-Schrieffer- (BCS) like interactions, ${ }^{27-30}$ dynamical channel blockades, ${ }^{31,32}$ and inelastic scattering. ${ }^{33}$ When a pair of Majorana bound states are coupled to two electron reservoirs directly, positive cross correlation may be induced by the nonlocal Andreev reflection, which means the injection of an electron into one bound state followed by the emission of a hole by the other. ${ }^{34}$
However, if two Majorana fermions are well separated, the nonlocal cross correlation might be absent, ${ }^{13,14,34,35}$ which implies that it is hard to obtain the nonlocal signal of Majorana fermions by electrical measurements. This would become a serious obstacle for the application of Majorana fermions in universal decoherence-free quantum computing. Furthermore, unlike Cooper pairs, in which the correlation is limited by the superconducting coherence length, ${ }^{27-29}$ the correlation between two Majorana fermions is not restricted by their separation in space. In such a real nonlocal system, nonlocal noise cross correlation is expected to be much more important than local current autocorrelations such as shot noise. ${ }^{25}$ Therefore, it is of fundamental interest to examine the noise cross correlation of two Majorana fermions in a long distance limit.

In this paper, we propose a way to generate nonlocal current noise cross correlation mediated by a pair of Majorana bound states. Differing from a system where the Majorana bound states couple to electron reservoirs directly, ${ }^{13,14,34}$ two quantum dots with single energy level are inserted between the Majorana bound states and reservoirs. In this case, local Andreev reflections involving a single reservoir can be suppressed by weak dot-reservoir coupling, while crossed Andreev reflections which split a Cooper pair over two reservoirs become dominant by strong coupling between the Majorana bound states and quantum dots. By tuning the gate voltages applied to the two dots, either positive or negative nonlocal cross correlations can be induced in this hybrid system of quantum dots and Majorana bound states. An experimental observation of the nonlocal noise correlation can serve as an alternative proof for the nonlocality of Majorana bound states.

The paper is organized as follows. In Sec. II, we propose the model system for the double quantum dots and Majorana bound states and introduce the rate equation formulism employed to study the currents and their correlations. In Sec. III, we present the results for the noise cross correlation between the currents flowing through the double dots and discuss the underlying physical processes. We also consider the effects of thermal fluctuation and local Andreev reflections 

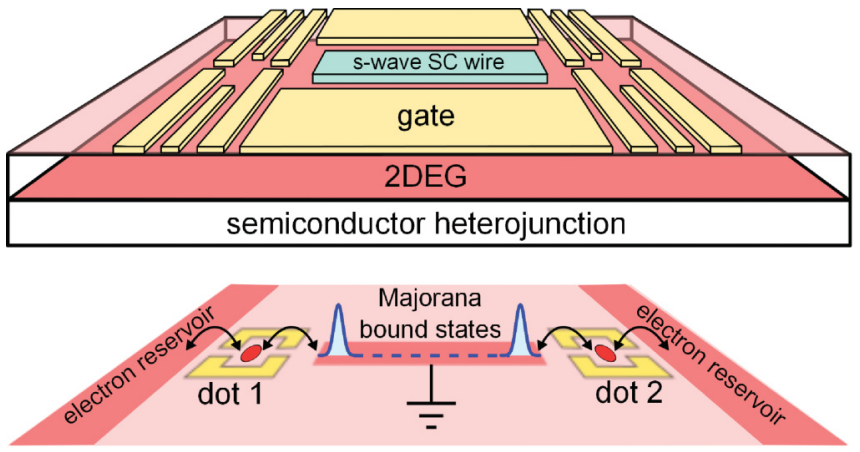

FIG. 1. (Color online) Setup with the double quantum dots and Majorana bound states. An $s$-wave superconductor wire is deposited on the surface of a semiconductor heterojunction, and a magnetic field is applied along the axis of the wire. For a proper magnetic field, two Majorana bound states appear at the ends of the topological superconductor. Two quantum dots are placed between the bound states and the reservoirs to block the local Andreev reflection between the Majorana bound states and electron reservoirs. The energy level of each dot can be modulated by applying a gate voltage.

enhanced by multiple dot levels. Finally, a summary is given in Sec. IV.

\section{MODEL AND FORMULISM}

\section{A. Setup}

Our proposed setup is sketched in Fig. 1. A two-dimensional electron gas at a semiconductor heterojunction, which is intensively studied in the context of both spin-orbit coupling and quantum dot, serves as the host for both the Majorana bound states and the dots. In the central region, spin-orbit coupling and strong Zeeman splitting give rise to a spinless energy band, which resembles a $p+i p$ topological superconductor when adjacent to an $s$-wave superconductor wire due to the proximity effect. ${ }^{10-12}$ The nontrivial topological nature of the $p+i p$ superconductor wire demands a pair of Majorana bound states to emerge at the two ends. Two quantum dots can be fabricated by depleting the electrons beneath the gate electrodes. Each quantum dot is coupled to a Majorana bound state and connected with the electron reservoir on its side. Only one spinless energy level is considered in each quantum dot by assuming sufficiently large level spacing and strong Zeeman field.

\section{B. Hamiltonian}

The Hamiltonian for the system in Fig. 1 is given by

$$
\begin{aligned}
H_{0}= & \epsilon_{1} d_{1}^{\dagger} d_{1}+\epsilon_{2} d_{2}^{\dagger} d_{2}+\frac{i}{2} \epsilon_{M} \eta_{1} \eta_{2} \\
& +\left(\lambda_{1}^{*} d_{1}^{\dagger}-\lambda_{1} d_{1}\right) \eta_{1}+i\left(\lambda_{2}^{*} d_{2}^{\dagger}+\lambda_{2} d_{2}\right) \eta_{2},
\end{aligned}
$$

where $\epsilon_{i}$ is the energy level in quantum dot $i(i=1,2)$ and $d_{i}\left(d_{i}^{\dagger}\right)$ is the annihilation (creation) operator of electron. The quantum dots are coupled to the Majorana bound states with strength of $\lambda_{1}$ and $\lambda_{2} . \epsilon_{M}$ is the "coupling strength" between the Majorana fermions $\eta_{1}\left(=\eta_{1}^{\dagger}\right)$ and $\eta_{2}\left(=\eta_{2}^{\dagger}\right)$ in the Majorana bound states. The electron reservoirs and their coupling to the dots are described by the Hamiltonian

$$
H_{T}=\sum_{i k} \epsilon_{i, k} c_{i k}^{\dagger} c_{i k}+\sum_{i, k}\left(t_{i} c_{i k}^{\dagger} d_{i}+\text { H.c. }\right),
$$

where $\epsilon_{i, k}$ is the electron energy in the reservoir $i$ and $t_{i}$ is the tunneling amplitude.

It is helpful to replace the Majorana fermion operators by a fermion operator, $\eta_{1}=f+f^{\dagger}, \eta_{2}=i\left(f^{\dagger}-f\right)$, where $f^{\dagger}$ creates a nonlocal fermion and $f^{\dagger} f=0,1$ counts the occupation of the corresponding state. ${ }^{13,17}$ In the new representation, the Hamiltonian in the central region becomes ${ }^{36}$

$$
\begin{aligned}
H_{0}= & \epsilon_{1} d_{1}^{\dagger} d_{1}+\epsilon_{2} d_{2}^{\dagger} d_{2}+\epsilon_{M}\left(f^{\dagger} f-\frac{1}{2}\right) \\
& +\left[\lambda_{1}\left(f^{\dagger} d_{1}+f d_{1}\right)+\lambda_{2}\left(f^{\dagger} d_{2}-f d_{2}\right)+\text { H.c. }\right]
\end{aligned}
$$

The Hamiltonian $H_{0}$ can be solved in the space spanned by eight basis states $\left|n_{1} n_{2} p\right\rangle$, where $n_{i}=0,1$ is the electron occupation number in the quantum dot $i$. For the Majorana bound states, the particle number in a superconducting states is not conserved, but the parity $p$ can serve as the quantum number, with the values of even $(e)$ or odd $(o)$. For the double dots and Majorana bound states, the energy eigenvalues and eigenstates are given in two closed subspaces of definite parity. It is found that the states $|00 e\rangle,|10 o\rangle,|01 o\rangle$, and $|11 e\rangle$ form a closed even block, and other four states $|00 o\rangle,|10 e\rangle,|01 e\rangle$, and $|11 o\rangle$ form a closed odd block. The eigenstates of even parity are

$$
\left|e_{l}\right\rangle=a_{l}^{e}|00 e\rangle+b_{l}^{e}|10 o\rangle+c_{l}^{e}|01 o\rangle+d_{l}^{e}|11 e\rangle
$$

and those of odd parity are

$$
\left|o_{l}\right\rangle=a_{l}^{o}|00 o\rangle+b_{l}^{o}|10 e\rangle+c_{l}^{o}|01 e\rangle+d_{l}^{o}|11 o\rangle
$$

with $l=1,2,3,4$ and where $a, b, c, d$ are normalized superposition coefficients. For the two parities, their eigenequations are

$$
\mathbf{M}^{o(e)} \Psi_{l}^{o(e)}=E_{l}^{o(e)} \Psi_{l}^{o(e)},
$$

where

$$
\begin{aligned}
\mathbf{M}^{e} & =\left(\begin{array}{cccc}
-\frac{\epsilon_{M}}{2} & \lambda_{1} & -\lambda_{2} & 0 \\
\lambda_{1}^{*} & \epsilon_{1}+\frac{\epsilon_{M}}{2} & 0 & \lambda_{2} \\
-\lambda_{2}^{*} & 0 & \epsilon_{2}+\frac{\epsilon_{M}}{2} & \lambda_{1} \\
0 & \lambda_{2}^{*} & \lambda_{1}^{*} & \epsilon_{1}+\epsilon_{2}-\frac{\epsilon_{M}}{2}
\end{array}\right), \\
\mathbf{M}^{o} & =\left(\begin{array}{cccc}
\frac{\epsilon_{M}}{2} & \lambda_{1} & \lambda_{2} & 0 \\
\lambda_{1}^{*} & \epsilon_{1}-\frac{\epsilon_{M}}{2} & 0 & -\lambda_{2} \\
\lambda_{2}^{*} & 0 & \epsilon_{2}-\frac{\epsilon_{M}}{2} & \lambda_{1} \\
0 & -\lambda_{2}^{*} & \lambda_{1}^{*} & \epsilon_{1}+\epsilon_{2}+\frac{\epsilon_{M}}{2}
\end{array}\right),
\end{aligned}
$$

and $\Psi_{l}^{o(e)}=\left(a_{l}^{o(e)}, b_{l}^{o(e)}, c_{l}^{o(e)}, d_{l}^{o(e)}\right)^{\mathbf{T}}$.

In the absence of the electron tunneling between the quantum dots and reservoirs, there is no mixing between the states of different parities. The parity in the hybrid system of Majorana bound states and dots can be varied by one-particle sequential tunneling between the quantum dots and the reservoirs. Thus, the even- and odd-parity sections are, 
thus, connected as

$$
\begin{aligned}
& \left\langle e_{l}\left|d_{1}\right| o_{l^{\prime}}\right\rangle=a_{l}^{e *} b_{l^{\prime}}^{o}+c_{l}^{e *} d_{l^{\prime}}^{o}, \\
& \left\langle e_{l}\left|d_{1}^{\dagger}\right| o_{l^{\prime}}\right\rangle=b_{l}^{e *} a_{l^{\prime}}^{o}+d_{l}^{e *} c_{l^{\prime}}^{o},
\end{aligned}
$$

for the tunneling events through dot 1 and

$$
\begin{aligned}
& \left\langle e_{l}\left|d_{2}\right| o_{l^{\prime}}\right\rangle=a_{l}^{e *} c_{l^{\prime}}^{o}+b_{l}^{e *} d_{l^{\prime}}^{o}, \\
& \left\langle e_{l}\left|d_{2}^{\dagger}\right| o_{l^{\prime}}\right\rangle=c_{l}^{e *} a_{l^{\prime}}^{o}+d_{l}^{e *} b_{l^{\prime}}^{o},
\end{aligned}
$$

for dot 2. The dot-reservoir tunneling does not mix the states of the same parity, i.e., $\left\langle e_{l}\left|d_{1(2)}^{\dagger}\right| e_{l^{\prime}}\right\rangle=0$ and $\left\langle o_{l}\left|d_{1(2)}^{\dagger}\right| o_{l^{\prime}}\right\rangle=0$.

\section{Rate equations}

We study the tunneling currents and their noise cross correlation in the sequential tunneling regime with the help of the rate equation method. ${ }^{37}$ Formally, there are two types of approaches: the generalized master equations and diagonalized rate equations. In the generalized master equations, the density matrix contains not only the population of each state (may not be the eigenstates) but also the coherent terms between any two states. The diagonalized rate equation approach is to, first, diagonalize the Hamiltonian of central region and then write down the rate equations for the population of central eigenstates. For weak coupling between the dots and reservoirs, it is safe to construct the density matrix $\rho$ with the eigenstates of the hybrid system of Majorana bound states and dots then write down the rate equations for the population of the system eigenstates. It has been shown that when the inner interaction of the central region is much stronger than the dot-lead coupling strength, the results obtained from two approaches are in close agreement with each other. ${ }^{37,38}$ Therefore, we take $\lambda_{i} \gg t_{i}$ in the calculation to ensure that the diagonalized rate equations work well. The time evolution of the density matrix $\rho(t)$ is given by the rate equations

$$
d \rho(t) / d t=\mathbf{W} \rho(t),
$$

where the rate matrix elements are $\mathrm{ar}^{37,39}$

$$
\begin{gathered}
W_{l^{\prime} l}=\sum_{i} \Gamma_{i}\left[f\left(\Delta_{l^{\prime} l}+\mu_{i}\right)\left|\left\langle l^{\prime}\left|d_{i}\right| l\right\rangle\right|^{2}\right. \\
\left.+f\left(\Delta_{l^{\prime} l}-\mu_{i}\right)\left|\left\langle l^{\prime}\left|d_{i}^{\dagger}\right| l\right\rangle\right|^{2}\right]
\end{gathered}
$$

for $l \neq l^{\prime}$ and

$$
W_{l l}=-\sum_{l^{\prime} \neq l}^{N} W_{l^{\prime} l},
$$

with $l, l^{\prime} \in\left\{\left|o_{l}\right\rangle,\left|e_{l}\right\rangle\right\}$. Here $f(\omega)=\left[1+e^{\omega / k_{B} T}\right]^{-1}$ is the Fermi distribution function, $\Delta_{l^{\prime} l}=E_{l^{\prime}}-E_{l}$ is the Bohr frequency of the transition from $|l\rangle$ to $\left|l^{\prime}\right\rangle, E_{l}$ is the eigenenergy of $|l\rangle$, and $\mu_{i}$ is the chemical potential in reservoir $i$. In the wide-band limit approximation, the coupling between the $i$-th dot level and its reservoir is denoted by $\Gamma_{i}=2 \pi\left|t_{i}\right|^{2} v_{i}$ with $v_{i}$ the spinless density of states near the Fermi surface of reservoir $i$. The current $I_{i}$ flowing through $\operatorname{dot} i$ is calculated by

$$
I_{i}=e \sum_{l}\left[\hat{\Gamma}^{i} \rho\right]_{l},
$$

where $\hat{\Gamma}^{i}$ is the matrix form of the current operator and its elements are given by

$$
\hat{\Gamma}_{l^{\prime} l}^{i}=\Gamma_{i}\left[f\left(\Delta_{l^{\prime} l}+\mu_{i}\right)\left|\left\langle l^{\prime}\left|d_{i}\right| l\right\rangle\right|^{2}-f\left(\Delta_{l^{\prime} l}-\mu_{i}\right)\left|\left\langle l^{\prime}\left|d_{i}^{\dagger}\right| l\right\rangle\right|^{2}\right] .
$$

The noise cross correlation of two tunneling currents can be studied by the Fourier transform of the current-current correlation function ${ }^{25}$

$$
S_{12}(\omega)=2 \int_{-\infty}^{\infty} d t e^{i \omega t}\left(\left\langle I_{1}(t) I_{2}(0)\right\rangle-\left\langle I_{1}\right\rangle\left\langle I_{2}\right\rangle\right),
$$

where $\langle\cdots\rangle$ represents the thermodynamic average. Furthermore, the current-current correlation function of the currents $I_{1}$ and $I_{2}$ in the $\omega$ space can be expressed as

$$
\left\langle I_{1}(t) I_{2}(0)\right\rangle_{\omega}=\sum_{k}\left[\hat{\Gamma}_{1} \hat{T}(\omega) \hat{\Gamma}_{2} \rho^{(0)}+\hat{\Gamma}_{2} \hat{T}(-\omega) \hat{\Gamma}_{1} \rho^{(0)}\right]_{k},
$$

where $\hat{T}( \pm \omega)=(\mp i \omega \mathbf{I}-\mathbf{W})^{-1}$ and $\mathbf{I}$ is the unit matrix.

In the following discussion, we focus on the situation that the two Majorana fermions are well separated so that $\epsilon_{M}=0$. The two dots are assumed symmetrically coupled to the reservoirs $\Gamma_{i}=\Gamma_{0}$. Considering the symmetric couplings between Majorana bound states and dots on the opposite ends, we take $\lambda_{i}=\lambda_{0}$.

\section{RESULTS AND DISCUSSIONS}

\section{A. Tunable nonlocal noise cross correlation}

The zero-frequency noise cross correlation $S_{12}$ is presented in Fig. 2 as a function of dot levels $\epsilon_{1}$ and $\epsilon_{2}$, where the voltage $V=-\mu_{i}$ is symmetrically applied to both dots. The correlation shows a quadrupole pattern of four regions, two positive and two negative. At the point $\epsilon_{1}=\epsilon_{2}=0$, the cross correlation is zero. Away from this point, the cross correlation with tunable signs can be induced by varying the dot energy levels. Along the diagonal directions, the correlation reaches the maxima. When both quantum dot levels are higher or lower than the chemical potential (set as zero energy point) in the $p+i p$ superconductor, the correlation is negative. Otherwise, the correlation is positive. Similar patterns were also obtained in the capacitively coupled double dots, ${ }^{32}$ where the cross correlation arises from the competition between the inter- and intradot Coulomb interactions. Here the sign reversal of the cross correlation is mediated by the Majorana bound states. Competition and coordination between different tunneling channels are modulated by the occupation of Majorana bound states. As shown in Fig. 2(a), two negative cross-correlation branches lie in the competition region asymmetrically and the maximum negative $S_{12}$ appears at the point $\epsilon_{i}=\mu_{i}$. Away from this point, electron tunneling into (out of) the dots becomes easier when $\epsilon_{i}<(>) \mu_{i}$. This weakens the competition between tunneling currents on the opposite ends and results in the decrease of noise cross correlation. 


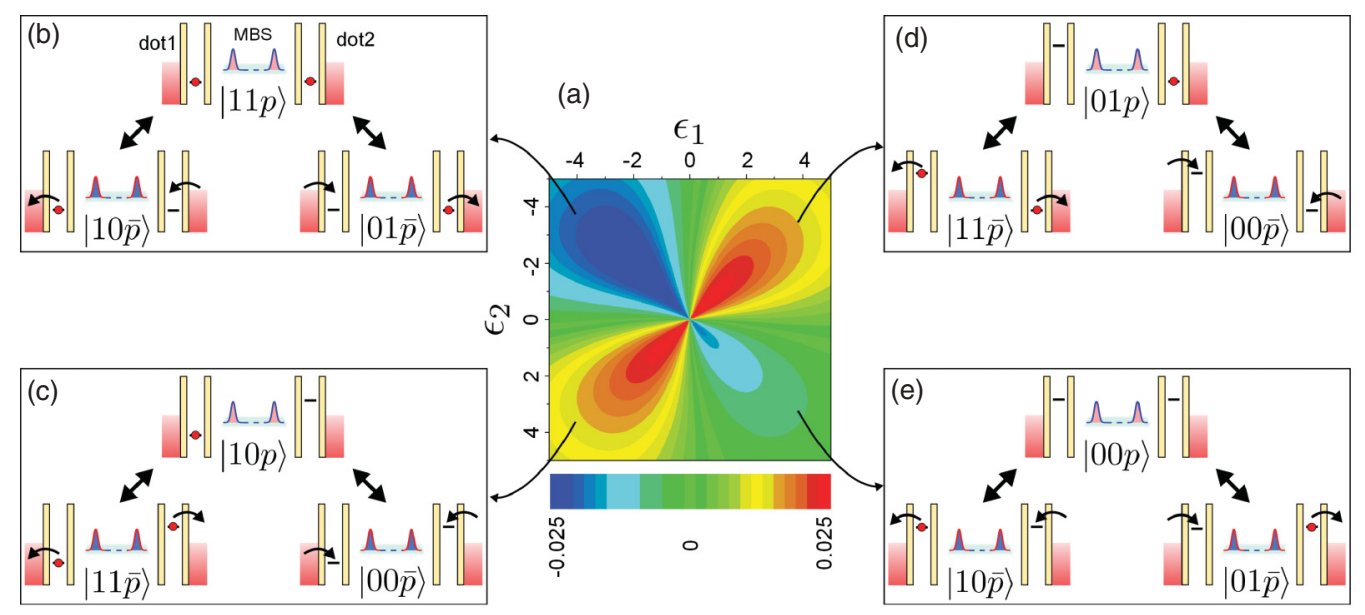

FIG. 2. (Color online) Tunable noise cross correlation. (a) Zero-frequency current noise cross correlation as a function of two dot energy levels $\epsilon_{1}$ and $\epsilon_{2}$. The parameters are taken as $\epsilon_{M}=0.0, k_{B} T=2.0, \Gamma_{0}=0.2, \lambda_{0}=5.0$, and $\mu_{i}=-2.0$. (b) When $\epsilon_{1}, \epsilon_{2}<0$, the system tends to reside in the state $\left|n_{1} n_{2} p\right\rangle=|11 p\rangle$, where $n_{i} \in\{0,1\}$ is the occupation in dot $i=1,2$, the parity $p$ of the Majorana bound states could be even or odd. $|11 p\rangle$ could evolve into either $|01 \bar{p}\rangle$ or $|10 \bar{p}\rangle$, where $\bar{p}$ stands for the opposite parity to $p$. For both $|01 \bar{p}\rangle$ and $|10 \bar{p}\rangle$, electron tends to tunnel in one dot and out of the other. These opposite tunneling processes lead to the negative cross correlation. Similarly, the correlation is also negative in (e) when $\epsilon_{1}, \epsilon_{2}>0$. (c) when $\epsilon_{1}<0<\epsilon_{2}$, the possible transitions are $|10 p\rangle \leftrightarrow|00 \bar{p}\rangle,|11 \bar{p}\rangle$, which tend to synchronize the tunnelings in two dot-reservoir channels and give rise to the positive correlation. Similar processes also give the positive cross correlation in (d) when $\epsilon_{2}<0<\epsilon_{1}$.

\section{B. Physical processes}

To analyze the physical processes beneath the correlation, we return to the $f$ representation in Eq. (3), which shows three types of transitions between the Majorana bound states and quantum dots: pairing creation $f^{\dagger} d_{i}^{\dagger}$, pairing annihilation $f d_{i}$, and direct tunneling $f^{\dagger} d_{i}$ and $d_{i}^{\dagger} f$. We, first, take Fig. 2(b), for example. By tuning the gate voltages, one can lower the energy levels in both dots than the chemical potentials in the reservoirs $\left(\epsilon_{i} \ll \mu_{i}\right)$. Consequently, the two dots are occupied most of the time and the system is in the state $|11 p\rangle$ (where $p$ can be even $e$ or odd $o$ ). In this state, thermal fluctuations can induce tunneling currents in both dot-reservoir channels, but the currents are uncorrelated when they are not related to the Majorana bound states. For $\epsilon_{i} \ll \mu_{i}$, the state population of $|00 p\rangle$ is rather small and almost does not participate in the state evolution. For weak dot-reservoir coupling, the states can evolute many times before parity is changed by an electron tunneling into or out of the reservoirs. The state $|11 p\rangle$ can evolve into $|10 \bar{p}\rangle$ or $|01 \bar{p}\rangle$ by direct tunneling if $p=e$ or pairing annihilation if $p=o$, where $\bar{p}=e$ if $p=o$ and vice versa. In either case, the occupations in the two dots change from $|11\rangle$ into $|10\rangle$ or $|01\rangle$. As a result of the interaction between the dots and Majorana bound states, electrons tend to tunnel into one dot and out of the other. These opposite tunneling behaviors lead to the negative cross correlation between the currents of two dot-reservoir channels. Similarly, for the case $\epsilon_{i} \gg \mu_{i}$ [Fig. 2(e)], the initial state $|00 p\rangle$ can evolve into $|10 \bar{p}\rangle$ or $|01 \bar{p}\rangle$, which also gives rise to the negative cross correlation. In contrast, when the zero energy point in the region of Majorana bound states is much higher than one dot energy level while much lower than the other, the possible transitions become from $|10 p\rangle$ to $|00 \bar{p}\rangle$ or $|11 \bar{p}\rangle$, as shown in Fig. 2(c), and from $|01 p\rangle$ to $|00 \bar{p}\rangle$ or $|11 \bar{p}\rangle$, as shown in Fig. 2(d). These transitions tend to synchronize the electron tunneling processes in both dots and make the cross correlation positive.

It is indicated in Fig. 2(a) that the noise cross correlation $S_{12}$ reaches the maximum values but with opposite signs on the two diagonal lines $\epsilon_{1}=\epsilon_{2}$ and $\epsilon_{1}=-\epsilon_{2}$. In these two cases, we can understand the nonlocal correlation from analytical results. In the case $\epsilon_{1}=\epsilon_{2}=\epsilon_{0}$, the eigenvalues of $H_{0}$ can be found as

$$
\begin{gathered}
E_{1 \pm}^{p}=\frac{1}{2}\left(3 \epsilon_{0} \pm \sqrt{\epsilon_{0}^{2}+8 \lambda_{0}^{2}}\right), \\
E_{2 \pm}^{p}=\frac{1}{2}\left(\epsilon_{0} \pm \sqrt{\epsilon_{0}^{2}+8 \lambda_{0}^{2}}\right),
\end{gathered}
$$

and the corresponding eigenstates are

$$
\begin{aligned}
& \Psi_{1 \pm}^{p}=\varsigma|10 \bar{p}\rangle+|01 \bar{p}\rangle+\frac{\epsilon_{0} \pm \sqrt{\epsilon_{0}^{2}+8 \lambda_{0}^{2}}}{2 \lambda_{0}}|11 \bar{p}\rangle, \\
& \Psi_{2 \pm}^{p}=\frac{\epsilon_{0} \mp \sqrt{\epsilon_{0}^{2}+8 \lambda_{0}^{2}}}{2 \lambda_{0}}|00 \bar{p}\rangle-|10 \bar{p}\rangle+\varsigma|01 \bar{p}\rangle,
\end{aligned}
$$

where $\varsigma=1$ for even parity and $\varsigma=-1$ for odd parity. When a relatively small voltage is applied to both dots, the eigenstate $\Psi_{1-}^{o(e)}\left(\Psi_{2-}^{o(e)}\right)$ is the ground state in the region of Majorana bound states and dots if the dot energy levels are lower (higher) than the chemical potential in the nanowire, i.e., $\epsilon_{0}<(>) 0$. For $\epsilon_{0}<0$, the stationary population of $\Psi_{1-}^{o(e)}$ is dominant and the transition between these two degenerate states could be caused by the tunneling between dots and reservoirs. When there is no interaction between the Majorana bound states and dots, the ground state is the one that the each dot is always singly occupied most of the time. The dot-Majorana interaction correlates the two dots nonlocally and induces the transition events $|11 p\rangle \leftrightarrow|01 \bar{p}\rangle$ or $|10 \bar{p}\rangle$. This means that the role of the coupling between the Majorana bound states and dots is to turn 
the same state in the two dots into opposite simultaneously. Therefore, the tunneling events between two channels tend to repulse each other, which gives rise to a negative noise cross correlation. Similarly, the ground state of the system is $\Psi_{2-}^{o(e)}$ in the case of $\epsilon_{0}>0$. The interaction between the Majorana bound states and dots causes the transition events $|00 p\rangle \leftrightarrow|01 \bar{p}\rangle$ or $|10 \bar{p}\rangle$. The noise cross correlation is also negative in this case.

When the two dot levels have opposite energies $\epsilon_{1}=-\epsilon_{2}=$ $\epsilon_{0}$, the eigenvalues of $H_{0}$ are

$$
\begin{gathered}
E_{1 \pm}^{p}=\frac{1}{2}\left(-\epsilon_{0} \pm \sqrt{\epsilon_{0}^{2}+8 \lambda_{0}^{2}}\right), \\
E_{2 \pm}^{p}=\frac{1}{2}\left(\epsilon_{0} \pm \sqrt{\epsilon_{0}^{2}+8 \lambda_{0}^{2}}\right),
\end{gathered}
$$

and the corresponding eigenstates are

$$
\begin{aligned}
& \Psi_{1 \pm}^{p}=\varsigma|00 \bar{p}\rangle+\frac{\epsilon_{0} \mp \sqrt{\epsilon_{0}^{2}+8 \lambda_{0}^{2}}}{2 \lambda_{0}}|01 \bar{p}\rangle-|11 \bar{p}\rangle, \\
& \Psi_{2 \pm}^{p}=|00 \bar{p}\rangle+\frac{\epsilon_{0} \pm \sqrt{\epsilon_{0}^{2}+8 \lambda_{0}^{2}}}{2 \lambda_{0}}|10 \bar{p}\rangle+\varsigma|11 \bar{p}\rangle .
\end{aligned}
$$

It is clearly shown that the coupling between the Majorana bound states and dots turns the opposite states of double dots into the same simultaneously, e.g., $|01 p\rangle(|10 p\rangle) \leftrightarrow|00 \bar{p}\rangle$ or $|11 \bar{p}\rangle$. The presence of Majorana-dot coupling tends to correlate the tunneling events in both dots in a bunching way. Therefore, the noise cross correlation is always positive in this case.

\section{Effects of thermal fluctuation}

In the sequential tunneling regime, the effect of thermal fluctuation is reflected in the Fermi distribution of tunneling electrons in both leads. The reservoirs are not affected by the coupling to the dots so they remain in their respective thermal equilibrium. With the decrease of temperature, fewer electrons away from the Fermi energy contribute to the current and thermal fluctuation-induced tunneling is suppressed. Figure 3 shows that the noise cross correlation $S_{12}$ exhibits quite different patterns for different temperatures $k_{B} T$. At low temperatures, the decay time of the lead correlation is mainly determined by the bias voltage, while the contribution from the quasiparticle lifetime is irrelevant. ${ }^{40,41}$ In the calculation, we take a bias voltage much larger than the dot-reservoir strength, which ensures that the results at relatively low temperatures are still justified. Figure 3(a) represents the results when the temperature is lower than the dot-lead coupling strength. In the region of negative values, the cross correlation decreases to zero for $\epsilon_{i}>0$ and shrinks to an elliptical region centering at $\epsilon_{0}=\mu_{i}$. Considered individually, the Majorana-dot Hamiltonian $H_{0}$ has particle-hole symmetry. However, when the reservoirs with tunable chemical potentials are connected to the dot, particle-hole symmetry is broken. In the calculation, we take the chemical potential in both leads $\mu=-2.0$ to generate two currents. Here the broken particle-hole symmetry is attributed to the finite bias voltage and the cross correlations thus differ in the regimes of $\epsilon_{i}>0$ and $\epsilon_{i}<0$. With the increase of temperature, four regions in the cross-correlation pattern become wider and more symmetric about the point
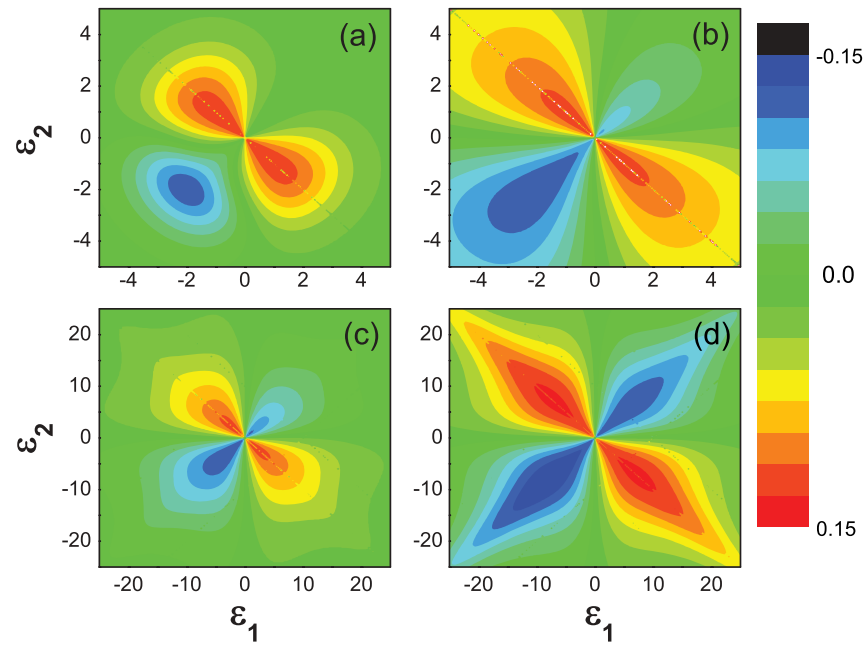

FIG. 3. (Color online) The current noise cross correlation as a function of two dot energy levels $\epsilon_{1}$ and $\epsilon_{2}$ for different temperatures $k_{B} T$. (a) $k_{B} T=0.5$, (b) $k_{B} T=2.0$, (c) $k_{B} T=5.0$, and (d) $k_{B} T=$ 10.0. The parameters are taken as $\Gamma_{0}=1.0, \lambda_{0}=5.0$, and $\mu_{i}=-2.0$.

$(0,0)$. From Eq. (10) it is found that the tunneling probability of electrons is governed by the Fermi distribution function $f\left(\Delta_{l^{\prime} l} \pm \mu_{i}\right)$. When $k_{B} T$ is much larger than $\lambda_{0}$, the tunneling probability is determined by the ratio $\epsilon_{i} / k_{B} T$. Therefore, the area of cross correlation is proportional to the energy scale of temperature approximately, as shown in Fig. 3.

It is interesting that the temperature does not suppress the strength of cross correlation, which differs from the case of capacitively coupled double dots. ${ }^{32}$ For interdot Coulomb interaction-induced cross correlations in double dots, each dot is connected to two electron reservoirs. At a relatively high temperature, the thermal fluctuation helps the electrons tunnel into or out of the dots more easily. The (anti-)blockade effect between two tunneling channels is, thus, weakened, leading to a suppression of noise cross correlation. However, for the present device, one of the reservoirs is replaced by one end of the topological superconductor. The zero-mode Majorana fermions serve as the source or drain, in which only one fermionic level takes part in the transport. Although the temperature determines the state population of the system, the mechanism of Majorana mediated cross correlation is independent of the temperature.

\section{Suppression of nonlocal noise correlation by enhanced local Andreev reflection via multiple dot levels}

In the setup where the Majorana bound states couple directly to the reservoirs, the noise cross correlation disappears ( $S_{12}=0$ ) for vanishing $\epsilon_{M} \cdot{ }^{13,14,34}$ Strictly speaking, $\epsilon_{M}$ must be 0 according to the definition that the Majorana fermions are their own antiparticles. In the present device the direct couplings between the Majorana bound states and reservoirs are blocked by the quantum dots. The weak dot-reservoir coupling suppresses the local Andreev reflection between the reservoirs and Majorana bound states and enhances the nonlocal cross correlation. To test this picture, we also study two cases in which multiple dot energy levels are coupled to the Majorana bound states: (1) only one end of the topological 
(a)

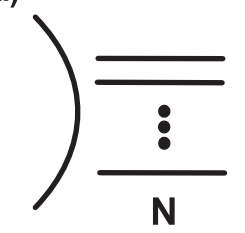

(b)
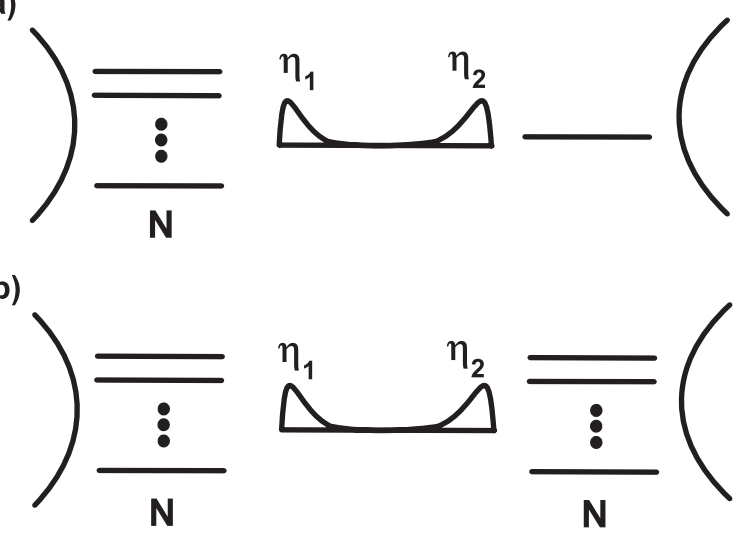

FIG. 4. Schematic view for the cases when multiple levels are coupled to the Majorana bound states at the ends of the topological superconductor. Multiple levels are coupled to (a) only one end of the topological superconductor and (b) both ends. Each level is assumed to have the same energy and coupling to the Majorana bound states.

superconductor is coupled to multiple levels and (2) both ends are coupled to multiple levels. The two cases are schematically shown in Figs. 4(a) and 4(b), respectively. The energy levels provide more conducting channels between the reservoirs and Majorana bound states, thus enhancing the local Andreev reflection. As the number $N$ of the energy levels approaches infinity, the model becomes equivalent to the case when the Majorana bound states are coupled to electron reservoirs directly. As a result, the current noise cross correlation is expected to vanish.

Figure 5 demonstrates the noise cross correlation $S_{12}$ as a function of the energy level $\epsilon_{d}$ for different level numbers $N$. For simplicity, it is assumed that the levels are of the same energy and coupling strength to the Majorana bound states. For comparison, Fig. 5(a) presents the result for the first case in Fig. 4(a), in which only one end couples to multiple levels, while Fig. 5(b) gives the result of the second case in Fig. 4(b) where both ends couple to multiple levels. For $N=1$, the model reduces to the case along $\epsilon_{1}=\epsilon_{2}$ in Fig. 2. In this case, the maximum negative $S_{12}$ can be achieved. It is found that with increasing number of energy levels, the cross correlation $S_{12}$ decreases rapidly in both cases. In the presence of multiple levels, the electron tunneling caused by Andreev reflection does not have to always involve both electron reservoirs but can occur via two channels in the same reservoir. In this way, the cross correlation between the currents flowing through two reservoirs is suppressed. Since the Andreev reflection can occur via any two levels, it is more likely to occur at the end of more energy levels for the case in Fig. 4(a). As a result, the suppression of $S_{12}$ by extra levels in Fig. 5(a) is much stronger than that in Fig. 5(b) as the level number $N$ increases.

\section{SUMMARY}

We propose a setup to generate nonlocal noise cross correlation between the currents flowing via two well-separated quantum dots, mediated by a pair of entangled Majorana fermions. Each dot is inserted between a Majorana bound state and a nearby electron reservoir to suppress the local Andreev reflection between them. In this case, the crossed
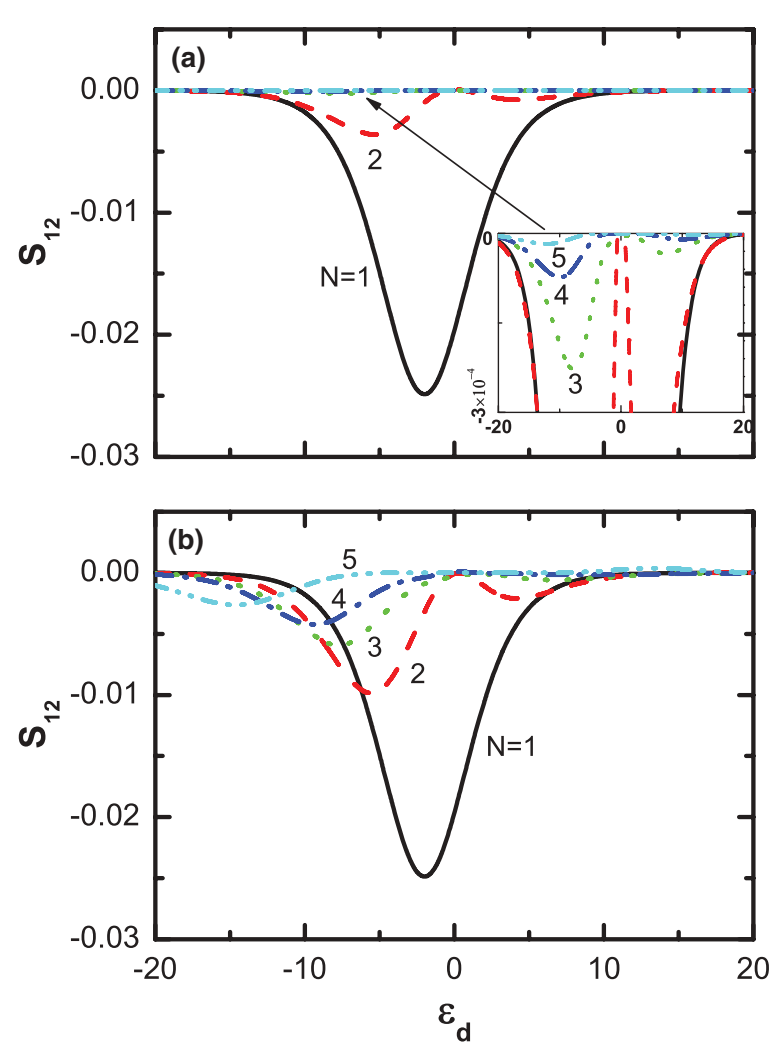

FIG. 5. (Color online) Current noise cross correlation as a function of energy level $\epsilon_{d}$ in the presence of multiple levels, where $N$ counts the level number. The $N$ levels couple to only one end (a) and both ends (b) of the topological superconductor, where the coupling strength between the $N$ levels and reservoirs is set as $\Gamma=\Gamma_{0} / N$. The parameters are taken as $\epsilon_{M}=0.0, k_{B} T=2.0, \Gamma_{0}=0.2, \lambda_{0}=10.0$, and $\mu_{i}=-2.0$.

Andreev reflection over two dots becomes dominant due to the strong coupling between the Majorana bound states and dots. It is demonstrated that the nonlocal cross correlation with tunable signs can be induced by the Majorana-dot coupling and modulated by the dot energy levels. Because the nonlocal correlation cannot be induced by other mechanisms, these measurable noise cross correlations can serve as a unique signature for the formation of Majorana bound states. It is found that the cross correlation is not suppressed with the increase of temperature and the area of correlation is proportional to the temperature approximately. Moreover, we study the case when multiple dot energy levels are coupled to the Majorana bound states. It is shown that with the increase of energy level number, the local Andreev reflection becomes enhanced and suppresses the crossed Andreev reflection considerably. As a result, the current noise cross correlation vanishes gradually.

\section{ACKNOWLEDGMENT}

We thank T. K. Ng and K. T. Law for insightful discussions. This project was supported by the Research Grant Council under Grant No. HKUST3/CRF/09, the Foundation for Innovative Research Groups of the NSFC under Grant No. 61021061, and the NSFC (Grants No. 11004022 and No. 61006081). 
${ }^{1}$ E. Majorana, Nuovo Cimento 14, 171 (1937).

${ }^{2}$ A. Stern, Nature (London) 464, 187 (2010).

${ }^{3}$ C. Nayak, S. H. Simon, A. Stern, M. Freedman, and S. Das Sarma, Rev. Mod. Phys. 80, 1083 (2008).

${ }^{4}$ G. Moore and N. Read, Nucl. Phys. B 360, 362 (1991).

${ }^{5}$ A. Kitaev, Ann. Phys. (NY) 303, 2 (2003).

${ }^{6}$ L. Fu and C. L. Kane, Phys. Rev. Lett. 100, 096407 (2008).

${ }^{7}$ J. D. Sau, R. M. Lutchyn, S. Tewari, and S. Das Sarma, Phys. Rev. Lett. 104, 040502 (2010).

${ }^{8}$ J. Alicea, Y. Oreg, G. Refael, F. von Oppen, and M. P. A. Fisher, Nat. Phys. 7, 412 (2011).

${ }^{9}$ J. Linder, Y. Tanaka, T. Yokoyama, A. Sudbø, and N. Nagaosa, Phys. Rev. Lett. 104, 067001 (2010).

${ }^{10}$ Y. Oreg, G. Refael, and F. von Oppen, Phys. Rev. Lett. 105, 177002 (2010).

${ }^{11}$ R. M. Lutchyn, J. D. Sau, and S. Das Sarma, Phys. Rev. Lett. 105, 077001 (2010).

${ }^{12}$ J. Alicea, Phys. Rev. B 81, 125318 (2010).

${ }^{13}$ C. J. Bolech and E. Demler, Phys. Rev. Lett. 98, 237002 (2007).

${ }^{14}$ K. T. Law, P. A. Lee, and T. K. Ng, Phys. Rev. Lett. 103, 237001 (2009).

${ }^{15}$ D. E. Liu and H. U. Baranger, Phys. Rev. B 84, 201308(R) (2011).

${ }^{16}$ A. Golub, I. Kuzmenko, and Y. Avishai, Phys. Rev. Lett. 107, 176802 (2011).

${ }^{17}$ M. Leijnse and K. Flensberg, Phys. Rev. B 84, 140501(R) (2011).

${ }^{18}$ M. Wimmer, A. R. Akhmerov, J. P. Dahlhaus, and C. W. J. Beenakker, New J. Phys. 13, 053016 (2011).

${ }^{19}$ K. Flensberg, Phys. Rev. Lett. 106, 090503 (2011).

${ }^{20}$ L. Fu, Phys. Rev. Lett. 104, 056402 (2010).

${ }^{21}$ V. Mourik, K. Zuo, S. M. Frolov, S. R. Plissard, E. P. A. M. Bakkers, and L. P. Kouwenhoven, Science 336, 1003 (2012).

${ }^{22}$ M. T. Deng, C. L. Yu, G. Y. Huang, M. Larsson, P. Caroff, and H. Q. Xu, arXiv:1204.4130.
${ }^{23}$ D. Goldhaber-Gordon, H. Shtrikman, D. Mahalu, D. AbuschMagder, U. Meirav, and M. A. Kastner, Nature (London) 391, 156 (1998).

${ }^{24}$ S. M. Cronenwett, H. J. Lynch, D. Goldhaber-Gordon, L. P. Kouwenhoven, C. M. Marcus, K. Hirose, N. S. Wingreen, and V. Umansky, Phys. Rev. Lett. 88, 226805 (2002).

${ }^{25}$ Y. M. Blanter and M. Büttiker, Phys. Rep. 336, 1 (2000).

${ }^{26}$ W. D. Oliver, J. Kim, R. C. Liu, and Y. Yamamoto, Science 284, 299 (1999).

${ }^{27}$ L. Hofstetter, S. Csonka, J. Nygård, and C. Schönenberger, Nature (London) 461, 960 (2009).

${ }^{28}$ J. Wei and V. Chandrasekhar, Nat. Phys. 6, 494 (2010).

${ }^{29}$ A. Freyn, B. Douçot, D. Feinberg, and R. Mélin, Phys. Rev. Lett. 106, 257005 (2011).

${ }^{30}$ P. Recher, E. V. Sukhorukov, and D. Loss, Phys. Rev. B 63, 165314 (2001).

${ }^{31}$ A. Cottet, W. Belzig, and C. Bruder, Phys. Rev. Lett. 92, 206801 (2004).

${ }^{32}$ D. T. McClure, L. DiCarlo, Y. Zhang, H. A. Engel, C. M. Marcus, M. P. Hanson, and A. C. Gossard, Phys. Rev. Lett. 98, 056801 (2007).

${ }^{33}$ C. Texier and M. Büttiker, Phys. Rev. B 62, 7454 (2000).

${ }^{34}$ J. Nilsson, A. R. Akhmerov, and C. W. J. Beenakker, Phys. Rev. Lett. 101, 120403 (2008).

${ }^{35}$ G. Strübi, W. Belzig, M. S. Choi, and C. Bruder, Phys. Rev. Lett. 107, 136403 (2011).

${ }^{36}$ S. Tewari, C. Zhang, S. Das Sarma, C. Nayak, and D. H. Lee, Phys. Rev. Lett. 100, 027001 (2008).

${ }^{37}$ C. Pöltl, C. Emary, and T. Brandes, Phys. Rev. B 80, 115313 (2009); H. Z. Lu, B. Zhou, and S. Q. Shen, ibid. 79, 174419 (2009).

${ }^{38}$ C. Emary, C. Pöltl, and T. Brandes, Phys. Rev. B 80, 235321 (2009).

${ }^{39} \mathrm{H}$. Bruus and K. Flensberg, Many-Body Quantum Theory in Condensed Matter Physics (Oxford University Press, Oxford, UK, 2004).

${ }^{40}$ A. Mitra and A. J. Millis, Phys. Rev. B 76, 085342 (2007).

${ }^{41}$ C. Timm, Phys. Rev. B 77, 195416 (2008). 